\title{
APLICAÇÃO DE FOTOIRRADIAÇÃO SOLAR PARA TRATAMENTO DE EFLUENTES DE LATICÍNIOS
}

\section{APPLICATION OF SOLAR PHOTOIRRADIATION FOR TREATMENT OF THE DAIRY EFFLUENT}

\author{
Jaqueline Luiza Maculan ${ }^{1}$ \\ Michel Luzza ${ }^{2}$ \\ Juan Carlos Pokrywiecki ${ }^{3}$ \\ Elisângela Dusmann ${ }^{4}$ \\ Ivane Benedetti Tonial ${ }^{5}$ \\ Ticiane Sauer Pokrywiecki ${ }^{6}$
}

\begin{abstract}
Resumo
A indústria de laticínios gera grande volume de efluentes. Estudos têm avaliado novas tecnologias para o tratamento destes efluentes, com destaque para os Processos Oxidativos Avançados. O presente estudo teve como objetivo avaliar a redução de carga orgânica de efluentes lácteos utilizando um processo fotocatalítico heterogêneo com a construção de um reator fotocatalítico $\left(\mathrm{TiO}_{2} / \mathrm{UV}\right.$ solar $)$. $\mathrm{O}$ reator foi montado em escala piloto para desenvolvimento dos testes preliminares e avaliação da efetividade do tratamento. Os efluentes foram avaliados nas formas: bruto (in natura) e tratado, exposto 2 horas à radiação solar com circulação do efluente sob a placa contendo $\mathrm{TiO}_{2}$. Foram avaliados as variáveis de $\mathrm{pH}$, cor, turbidez, DBO e DQO. Os resultados revelaram que houve aumento nos parâmetros: $\mathrm{pH}$, cor e turbidez do efluente tratado, no entanto, houve redução de $66,1 \%$ para a DBO e $66,5 \%$ para a DQO, indicando uma considerável diminuição de matéria orgânica no efluente. Porém, novos estudos deverão ser realizados com o objetivo de minimizar principalmente a cor e turbidez a fim de possibilitar melhoria no tratamento e possível utilização em escala industrial deste equipamento.
\end{abstract}

Palavras-chave: Efluente lácteo; Matéria Orgânica; Fotoirradiação solar.

\begin{abstract}
The dairy industry is considered among the food industry, one of the most polluting because it generates a large volume of effluents. At present studies have evaluated new technologies for the treatment of this type of effluent, especially the Advanced

\footnotetext{
${ }^{1}$ Discente do Curso de Especialização em Ciência e Tecnologia Ambiental da Universidade Tecnológica Federal do Paraná, Campus Francisco Beltrão-PR. Francisco Beltrão - Paraná. E-mail: jaque_maculan@hotmail.com

${ }_{2}^{2}$ Discente do Curso de Graduação em Engenharia Ambiental da Universidade Tecnológica Federal do Paraná, Campus Francisco Beltrão-PR. Francisco Beltrão - Paraná. E-mail: michelluzza@ gmail.com

${ }^{3}$ Docentes do Curso de Graduação em Engenharia Ambiental e do Curso de Especialização em Ciência e Tecnologia Ambiental da Universidade Tecnológica Federal do Paraná, Campus Francisco Beltrão-PR. Francisco Beltrão - Paraná. E-mail: Juan@utfpr.edu.br

${ }^{4}$ Docentes do Curso de Graduação em Engenharia Química da Universidade Tecnológica Federal do Paraná, Campus Francisco Beltrão-PR. Francisco Beltrão - Paraná. E-mail: edusman@ utfpr.edu.br

${ }^{5}$ Docentes do Curso de Graduação em Engenharia Ambiental e do Curso de Especialização em Ciência e Tecnologia Ambiental da Universidade Tecnológica Federal do Paraná, Campus Francisco Beltrão-PR. Francisco Beltrão - Paraná. E-mail: ivane@utfpr.edu.br

${ }^{6}$ Docentes do Curso de Graduação em Engenharia Ambiental e do Curso de Especialização em Ciência e Tecnologia Ambiental da Universidade Tecnológica Federal do Paraná, Campus Francisco Beltrão-PR. Francisco Beltrão - Paraná. E-mail: ticiane@utfpr.edu.br
} 
Oxidation Processes. This study aimed to evaluate the organic material reduction of dairy effluent using a heterogeneous photocatalytic process with the construction of a photocatalytic reactor $\left(\mathrm{TiO}_{2} / \mathrm{UV}\right.$ solar $)$. The reactor was mounted on a pilot scale for development of preliminary testing and evaluation of the effectiveness of treatment. The effluents were evaluated in the forms: raw (in natura) and treated, two hours exposed to solar radiation with circulation of effluent under the plate containing $\mathrm{TiO} 2$. Were evaluated the variables $\mathrm{pH}$, color, turbidity, BOD and COD. The results show that increasing $\mathrm{pH}$, color and turbidity of the effluent and a reduction of $66,1 \%$ to $66.5 \%$ for $\mathrm{BOD}$ and $\mathrm{COD}$, indicating a considerable reduction in organic material. However, new studies should be conducted in order to primarily minimize color and turbidity in order to provide improved treatment and possible use on an industrial scale of this equipment.

KEY WORDS: Dairy effluent; Organic material; Photoirradiation solar.

\section{INTRODUÇÃO}

A indústria de laticínios é considerada, dentre as indústrias alimentícias uma das mais poluentes, gerando um grande volume de efluentes, 2,5 litros de efluente para cada litro de leite processado, e se lançado ao ambiente sem o prévio tratamento compromete sua qualidade e saudabilidade (PUPO NOGUEIRA, 2007; VOURCH et al., 2008; GOBLOS et al., 2008).

Os efluentes da indústria de laticínios são caracterizados por apresentarem altos índices de DBO (Demanda Bioquímica de Oxigênio) e DQO (Demanda Química de Oxigênio), além de altos níveis de sólidos dissolvidos, gorduras e nutrientes (amônia e fósforo) (CORDI et al., 2008; SAMANAMUD, 2011).

Para minimização ou até mesmo redução da carga orgânica e microrganismos patogênicos, estas indústrias utilizam técnicas convencionais de tratamento biológico, que são caracterizadas por apresentarem boa eficiência, fácil operação, baixo custo e ser promissora para a redução da carga orgânica dos efluentes lácteos (COSTA et al., 2003; ALTURKMANI, 2006). No entanto, estes sistemas de tratamento podem apresentar desvantagens que comprometem sua eficiência, dentre as quais: possibilidade de geração de maus odores, longo tempo de tratamento, remoção ineficiente de nutrientes e patogênicos, sobrecarga dos sistemas biológicos de tratamento, grande geração de lodo, além de haver um consumo considerável de energia pela presença de aeradores (JANCZUKOWICZ et al., 2008; FAEDO, 2010).

Para suprir esta deficiência, surgem novas tecnologias, dentre os quais se citamos Processos Oxidativos Avançados (POAs). Estas técnicas se baseiam na utilização de espécies altamente oxidantes responsáveis pela degradação mais efetiva dos poluentes e quando empregado em conjunto com tratamentos biológicos reduzem o 
tempo de tratamento (DE MORAES e PERALTA-ZAMORA, 2005; BRITO e SILVA, 2012).

A grande vantagem desses processos é que ocorre a mineralização da grande maioria dos contaminantes orgânicos. Através de uma série de reações químicas os contaminantes são transformados em dióxido de carbono, água e ânions, ou seja, o composto não muda de fase, como ocorre na maioria dos processos de tratamento (SAUER, 2006).

Dentre os POAs, os processos de fotocatálise heterogênea, como o $\left(\mathrm{TiO}_{2} / \mathrm{UV}\right.$ solar) tem sido investigado para o tratamento de efluentes industrias. Trata-se de uma das mais importantes tecnologias de remoção de contaminantes em águas residuais (GARCÉS e PEÑUELA, 2005). Nesta técnica, o dióxido de titânio $\left(\mathrm{TiO}_{2}\right)$ é o catalisador mais utilizado na fotocatálise por apresentar algumas características entre as quais: não tóxico, baixo custo, insolubilidade em água, foto-estabilidade, estabilidade química em uma ampla faixa de $\mathrm{pH}$ e possibilidade de ativação pela luz solar, o que reduz os custos do processo (ALMEIDA, 2011; BRITO e SILVA, 2012). Além disso, o catalisador pode ser utilizado no sistema em suspensão ou na forma imobilizada (SAUER, 2006).

A utilização de fotoirradiação por luz solar na placa com $\mathrm{TiO}_{2}$ imobilizado tem demonstrado bons resultados com considerável redução nos valores de DQO e COT (carbono orgânico total) em efluentes lácteos e de destilaria de álcool (SALAZAR, 2009; CARROCCI, 2009).

Neste sentido, o objetivo deste estudo foi avaliar a redução de carga orgânica em efluentes de laticínios com aplicação de fotocatálise heterogênea $\left(\mathrm{TiO}_{2} / \mathrm{UV}_{\text {solar}}\right)$, comparando os resultados obtidos com o tratamento biológico convencional.

\section{MATERIAIS E MÉTODOS}

$\mathrm{O}$ reator piloto de aplicação fotocatálise heterogênea $\left(\mathrm{TiO}_{2} / \mathrm{UV}_{\text {volar }}\right)$ para tratamento de efluentes foi desenvolvido no laboratório de águas e resíduos líquidos da Universidade Tecnológica Federal do Paraná- Campus Francisco Beltrão (UTFPR-FB), conforme item 2.2.

\subsection{Amostragem}


O efluente utilizado para o teste foi coletado, em três repetições de 20 litros cada, em uma indústria de beneficiamento de leite, localizada no município de Francisco Beltrão-PR. Para isso foi utilizado o efluente bruto, ou seja, ausente de qualquer tipo de tratamento prévio.

Para manter as características do efluente bruto (in natura) após coleta este foi armazenado em frascos âmbar $-4^{\circ} \mathrm{C}$. As análises de caracterização físico-química foram realizadas antes e após o tratamento biológico convencional e antes e após o tratamento com reator solar (fotocatálise heterogênea utilizando $\mathrm{TiO}_{2}$ como catalizador) a fim de avaliar o percentual de remoção de poluentes com a aplicação da técnica.

\subsection{Reator solar: Montagem e mecanismo de funcionamento}

Para a degradação do efluente lácteo foi utilizado um sistema baseado em fotocatálise heterogênea com $\mathrm{TiO}_{2}$ fixo e radiação solar.

O sistema (reator solar) foi construído utilizando-se uma chapa metálica de $60 \times 36 \mathrm{~cm}$, com uma área útil de $2160 \mathrm{~cm}^{2}$. Esta chapa foi revestida com uma mistura de resina poliuretânica e $\mathrm{TiO}_{2}$. A tinta aplicada sobre as placas foi produzida com uma concentração de catalisador de $25,0 \%$. A aplicação da tinta na placa foi realizada de acordo com Salazar et al. (2009). Com ajuda de uma bomba centrífuga, o efluente era bombeado e distribuído uniformemente à chapa metálica recoberta com a mistura fotocatalítica $\left(\mathrm{TiO}_{2} / \mathrm{UV}\right)$ com vazão de escoamento de $0,25 \mathrm{~mL} \cdot \mathrm{min}^{-1}$ que exposto à luz solar para ação das radiações UV. Após a passagem pela placa, o efluente foi então coletado em um reservatório com capacidade para 5 litros e redirecionado ao tanque contendo o efluente bruto. Este ciclo foi repetido por 2 horas sendo, então, o efluente coletado para realização das análises.

O esquema que mostra o reator está apresentado na Figura 1.

Figura 1 - Esquema do reator fotocatalítico.

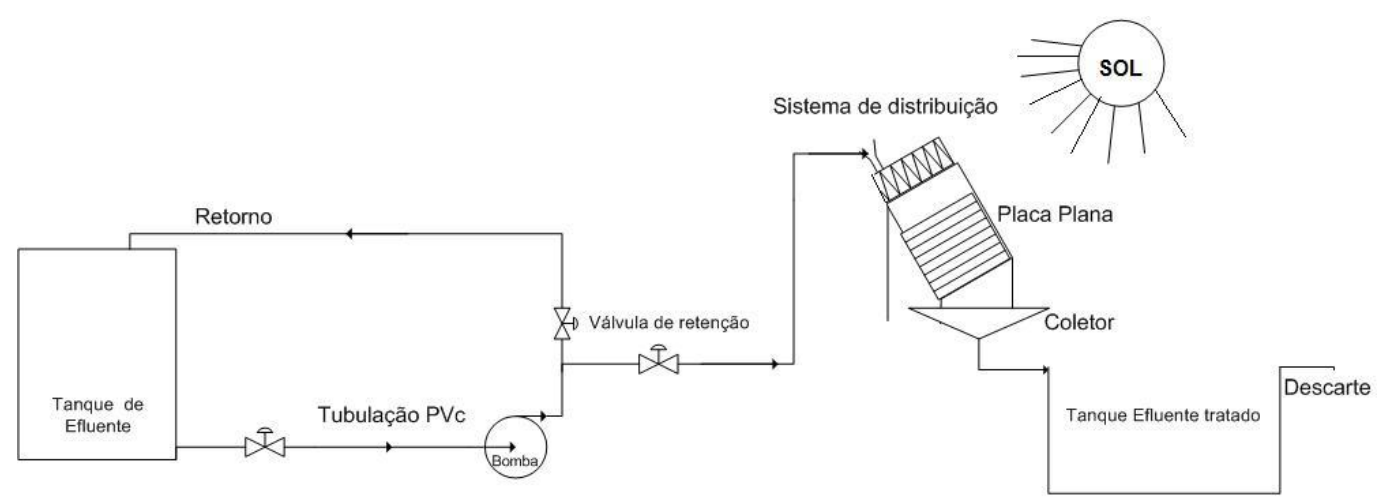




\subsection{Análises de caracterização do efluente bruto e tratado}

O efluente após tratamento biológico convencional e tratamento fotocatalítico foi coletado e encaminhado para a realização das análises de caracterização onde foram avaliados o pH, cor, turbidez, demanda bioquímica de oxigênio (DBO) e demanda química de oxigênio (DQO) no laboratório de Análises de água e Resíduos líquidos da UTFPR-FB. Todos os métodos de análise utilizados no presente estudo estão descritos no "Standard Methods" (APHA, AWWA e WEF, 1995).

\subsection{Avaliação do processo de tratamento}

A avaliação da eficiência da remoção de carga orgânica foi efetuada com base na equação a seguir, também adotada em estudo realizado por Koetz, Faria e Nunes (1996).

$\mathrm{E}=\left[\left(\mathrm{C}_{0}-\mathrm{C}_{\mathrm{f}}\right) \times 100 / \mathrm{C}_{0}\right]$

Sendo:

E: Eficiência de remoção em porcentagem (\%);

$\mathrm{C}_{0}$ - Valor de concentração inicial ou efluente bruto em mg. $\mathrm{L}^{-1}$;

$\mathrm{C}_{\mathrm{f}}$ - Valor de concentração final ou efluente tratado em mg. $\mathrm{L}^{-1}$.

\section{RESULTADOS E DISCUSSÃO}

Os parâmetros avaliados cor, turbidez, DQO, DBO e pH nos efluentes bruto e tratado, permitem uma comparação entre estes, permitindo a determinação percentual de redução de cada um das variáveis antes e após o tratamento. Assim, a Tabela 1 apresenta os resultados da caracterização para os efluentes láctico, bruto e tratado, bem como a porcentagem de redução de cada variável após o tratamento biológico convencional.

Tabela 1- Características dos efluentes de uma indústria de beneficiamento de produtos lácteos antes e após o tratamento biológico convencional.

\begin{tabular}{|c|c|c|c|}
\hline Parâmetros & E.B.* & E.T.** & \% de Remoção \\
\hline$\left.\overline{\text { Cor (uH mg Pt-Co.L }}{ }^{-1}\right)$ & 20 & 12 & 40,0 \\
\hline Turbidez (NTU) & 853 & 44,4 & 94,8 \\
\hline
\end{tabular}


Demanda Bioquimica de Oxigênio (mg. $\left.\mathrm{L}^{-1}\right) \quad 680$

Demanda Quimica de Oxigênio (mg.L $\mathrm{L}^{-1}$ )

$\mathrm{pH}$

* Efluente Bruto (In natura); **Efluente tratado (Tratamento convencional)

Os dados mostram que o percentual de redução para os parâmetros cor e turbidez foram de 40,0\% e 94,8\%, respectivamente, para o efluente após tratamento biológico convencional. A cor e a turbidez são parâmetros que estão diretamente relacionados entre si, considerando que ambos resultam da presença de partículas (no estado coloidal e suspensão) da matéria orgânica e inorgânica dissolvida, pois, de acordo com Begnini e Ribeiro (2014), o processo produtivo da indústria pode gerar grande quantidade de sólidos em suspensão o que pode potencializar o teor de turbidez, pois ambos se correlacionam diretamente.

A redução deste parâmetro se torna fundamental durante o tratamento dos efluentes, pois se a turbidez estiver alta existe uma maior dificuldade dos raios solares incidirem sobre o efluente, com isso os microorganismos fotossintetizantes também são prejudicados assim como a concentração de oxigênio (FERREIRA, 2008).

Observa-se (Tabela 1) que o tratamento biológico convencional proporcionou uma excelente redução na turbidez do efluente láctico $(\sim 95,0 \%)$, o que pode estar refletido na redução de sólidos após o tratamento, indicando que para este parâmetro, o tratamento aplicado foi eficiente.

Os resíduos industriais são considerados na atualidade potencias poluidores do ambiente, sendo exigida pela legislação brasileira a redução da carga poluidora, no caso de efluentes de indústrias alimentícias se faz menção à matéria orgânica, para seu posterior lançamento. Assim, com relação aos teores de DBO e DQO os percentuais de redução foram de $78,7 \%$ e $77,3 \%$ respectivamente. Os valores encontrados para os teores de DQO e DBO estão de acordo com o observado por outros autores, pois, este tipo de efluente apresenta alta carga orgânica (lipídeos, proteínas e carboidratos) e se lançados aos corpos hídricos colocam em risco o ecossistema aquático, pois, promovem a redução na concentração de oxigênio dissolvido (VILLA; SILVA; NOGUEIRA, 2007; FERREIRA, 2008).

Dados semelhantes (DQO - $1219 \mathrm{mgO}_{2} \cdot \mathrm{L}^{-1} ; \mathrm{DBO}-540 \mathrm{mgO}_{2} \cdot \mathrm{L}^{-1}$ ) aos encontrados no presente estudo foram obtidos por Machado et al. (2012) que avaliou o 
processo de degradação da matéria orgânica em diferentes etapas do processo de tratamento de efluente lácteo.

Os resultados referentes ao tratamento do efluente bruto utilizando o reator fotocatalítico por 120 minutos ( 2 horas) estão apresentados na Tabela 2.

Tabela 2 - Características do efluente antes e após o tratamento com reator fotocatalítico

\begin{tabular}{lccc}
\hline Parâmetros & E.B.* & E.T.** & \% de Remoção \\
\hline Cor $\mu$ H (mg Pt-Co.L-1) & 55 & 68 & $+23,6$ \\
pH & 6,29 & 6,60 & - \\
Turbidez (NTU) & 807 & 924 & $+14,5$ \\
DBO (mg.L-1) & 677 & 227 & 66,5 \\
DQO (mg.L-1) & 1415 & 479,0 & 66,1 \\
DQO/DBO & 2,09 & 2,11 & - \\
\hline
\end{tabular}

* Efluente Bruto (In natura); **Efluente tratado (Tratamento Fotocatalítico)

Os dados mostram que a DBO e a DQO obtiveram uma remoção de 66,5 e $66,1 \%$, respectivamente, indicando que o sistema fotocatalítico apresentou bons resultados na degradação da matéria orgânica, uma vez que o tempo de exposição do efluente ao reator e do índice de radiação UV foram baixos. Além disso, o valor de DBO atingiu a redução mínima de $60,0 \%$ que vem ao encontro com o estabelecido na Resolução do CONAMA 430/2011. O valor de pH apresentou pequena variação quando se compara o efluente bruto $(6,29)$ com o efluente tratado $(6,60)$. Resultados inferiores $(55,0 \%)$ para a de redução de DQO foram observados por Salazar (2009) em efluentes lácteos utilizando fotoirradição solar com placa de $\mathrm{TiO}_{2}$ por um tempo de 3 horas e por Carrocci (2009) (45,4\%) em pré-tratamento de vinhaça de destilaria de álcool, também com a utilização de fotoirradição solar com placa de TiO2.

Estes resultados quando comparados com os dados obtidos no tratamento convencional são relevantes, uma vez que a diferença entre os percentuais de redução dos tratamentos foram de aproximadamente $10,0 \%$. No tratamento convencional o valor de redução da carga orgânica (DQO) obtida foi de 77,3\% enquanto que por meio de fotocatálise a redução foi de $66,1 \%$ para remoção.

Apesar disto, para os dados de cor e turbidez, o tratamento do efluente lácteo com o reator fotocatalítico apresentou menor efetividade quando comparado ao 
tratamento biológico convencional, pois se observa (Tabela 2) um aumentou nos valores destes parâmetros após o tratamento fotocatalítico, o que possivelmente pode estar relacionado com o desprendimento de $\mathrm{TiO}_{2}$ da placa metálica. Apesar disto, os fatores tempo de tratamento e espaço físico devem ser considerados, e estes, favorecem a catálise heterogênea.

\section{CONCLUSÃO}

O presente estudo avaliou a degradação da matéria orgânica de efluentes lácteos mediante aplicação de processos oxidativos avançados (POAs) utilizando catálise heterogênea $\left(\mathrm{UV} / \mathrm{TiO}_{2}\right)$ em comparação ao tratamento biológico convencional. A diferença no percentual de remoção da carga orgânica pode ser considerada eficaz, uma vez que o tempo de exposição do reator e radiação UV - Solar foi de 2 horas, tempo este considerado pequeno quando comparado ao tempo necessário em uma ETE convencional. Os resultados mostraram que a aplicação fotocatalítica, contribuiu para redução no teor de matéria orgânica medida em DQO $(66,5 \%)$ e DBO $(66,1 \%)$, atingindo a porcentagem de remoção necessária para estar em conformidade com a legislação vigente, confirmando assim a eficiência do reator fotoquímico para o efluente utilizado.

\section{REFERÊNCIAS}

ALMEIDA, M. K. Fotocatálise Heterogênea Aplicada Na Degradação Do Corante Rodamina-B Utilizando Nanopartículas de Dióxido de Titânio. 2011. 97f. Dissertação (Mestrado em Tecnologia Ambiental). Universidade de Santa Cruz do Sul UNISC, Rio Grande do Sul. 2011.

ALTURKMANI, A. Anaerobic treatment of whey in stirred batch reactor. In: Dairy Industry Effluents treatment, p.1-8, 2006. Homs Dairy Company Publication. Disponível em: http//www.homs-dairy.com. Acesso em: 04 março 2015.

AWWA. Standard Methods for the Examination of Water and Wastewater. $19^{\text {th }}$ edition (1995). Publication Office American Public Health Association, Washington. APHA, AWWA, WEF.

BEGNINI, B.C.; RIBEIRO, H.B. Plano Para Redução De Carga Poluidora Em Indústria De Lacticínios. Saúde e Meio Ambiente, v. 3, n. 1, p. 19-30, jan./jun. 2014.

BRASIL. Resolução CONAMA 357 de 17 de março de 2005. Brasília, Distrito Federal, 2005.

BRASIL. Resolução CONAMA 430 de 13 de maio de 2011. Brasília, Distrito Federal, 2011. 
BRITO, N. N. De.; SILVA, V. B. M. Processos Oxidativos Avançados e sua aplicação ambiental. Revista Eletrônica de Engenharia Civil, n.3, v.1, p.36-47, 2012.

CARROCCI, J. A. Aplicação de catálise heterogênea com $\mathrm{TiO}_{2}$ fotoirradiada por luz solar como pré-tratamento de vinhaça para posterior tratamento biológico. 2009. 120f. Dissertação (Mestrado em Ciências) - Escola de Engenharia de Lorena EEL/USP, Lorena, São Paulo, 2009.

CORDI, S.; ASSALIN, M.R.; DIEZ, M.C.; DURAN, N. Montagem, partida e operação de um sistema de lodosativados para o tratamento de efluentes: parâmetros físicoquímicos e biológicos. Engenharia Ambiental. v.5, n.1, p. 97-115. 2008.

COSTA, L.L.; CEBAllos, B.S.O; MEIRA, C.M.B.S.; CAVALCANTI, M.L.F. Eficiência de Wetlands construídos com dez dias de detenção hidráulica na remoção de colifagenos e bacteriófogos. Revista de Biologia e Ciências da Terra. v.3, n.1, 2003.

DE MORAES, J. L.; PERALTA-ZAMORA, P. Use of advanced oxidation processes to improve the biodegradability of mature landfill leachates. Journal of Hazardous Materials, v.123, n.1-3, p. 181-186, 2005.

FAEDO, A. M. Tecnologias convencionais e novas alternativas para o tratamento de efluentes domésticos. 2010. 39p. Trabalho de conclusão de especialização (Curso de Engenharia do controle da poluição ambiental). Universidade do Sul de Santa Catarina, Florianópolis, 2010.

FERREIRA, D. G. Influência da aplicação de boas práticas de fabricação sobre a quantidade e qualidade do efluente bruto de uma indústria de Laticínios em Caldazinha-GO. 2008. 89f. (Mestrado em Engenharia Agrícola - Recursos Hídricos e Saneamento Ambiental) - Universidade Estadual de Goiás UEG, Anápolis, 2008.

GARCÉS, L.F.; PEÑUELA, G. A. "Cinética de degradación y mineralización del colorante Naranja Reactivo 84 en agua," Revista Lasallista de Investigación, v. 2, n.2, p. 21-25, 2005.

GOBLOS, S. S.; PORTORO, P., BORDÁS, D., KÁLMAN, M.;KISS, I. Comparison of the effectivities of two-phase and single-phase anaerobic sequencing batch reators during dairy wastewater treatment. Renew able Energy. v.33, p. 960-965, 2008.

JANCZUKOWICZ, W.; ZIELINSKI, M.; DEBOWSKI, M. Biodegradability evaluation of dairy effluents originated in selected sactions of dairy production. Bioresource Tecnology, v. 99, n. 10, p. 4199 - 4205, 2008.

KOETZ, P. R.; FARIA, O. L. V.; NUNES, W. A. Tratamento de efluentes da indústria de arroz parboilizado por digestão anaeróbia em reatores de fluxo ascendente. Revista Brasileira de Agrociência, v. 2, n. 2, p. 117-120, 1996.

MACHADO, E.L.; TERESINHA, K.T.L.; MEYER, K.R.O.; SCHNEIDER, L. Gestão tecnológica ambiental de uma Indústria de Laticínios: ênfase em efluentes. XMachado-Brasil-1. 2012.

PUPO NOGUEIRA, R. F., TROVÓ, A. G., SILVA, M. R. A., VIlla, R. D. Fundamentos e aplicações ambientais dos processos fenton e foto-fenton. Química Nova. v. 30, n.2, p. 400 - 408, 2007.

SALAZAR, R.F.S. Aplicação de processo oxidativo avançado (POA) como prétratamento de efluentes de laticínio para posterior tratamento biológico. 2009, 
210f. Dissertação (Mestrado em Engenharia Química) - Escola de Engenharia de Lorena EEL/USP, Lorena, São Paulo, 2009.

SAMANAMUD, G.R.L. Estudo da Aplicação de ZnO fotoirradiado com luz solar no tratamento de efluentes de laticícinos. 2011, 163f. Dissertação (Mestrado em Ciências). Escola de Engenharia de Lorena. Universidade de São Paulo, Lorena, São Paulo. 2011.

SAUER, T. Tratamento de efluentes de curtume através do processo combinado de degradação fotocatalítica seguida por adsorção em carvão ativado. 2006, 249f. Tese (Doutorado em Engenharia Química) - Universidade Federal de Santa Catarina, Florianópolis, Santa Catarina. 2006.

VILLA, R.D; SILVA, M.R. A.; NOGUEIRA, R. F. P. Potencial de aplicação do processo foto fenton/solar como o pré-tratamento de efluente da indústria de laticínios. Química Nova, v.30, n.8, p. 1799-1803, 2007.

VOURCH, M.; BALANNEC, B.; CHAUFER, B.; DORANGE, G. Treatment of dairy industry wastewater by reverse osmosis for water reuse. Desalination, v. 219, p. 190202, 2008. 